\title{
REVIEW
}

\section{Update on osteoarthritis part 1: current concepts and the relation to exercise}

\section{P G Conaghan}

Br J Sports Med 2002;36:330-333

There appears to be an increased risk of lower limb osteoarthritis in participants of repetitive, high impact sports, and this is strongly associated with joint injury. There seems to be little risk associated with recreational running. Assessment of risk for osteoarthritis should take into account the nature of the sport, intensity of training, presence of previous injury, body mass index, and occupation.
Correspondence to: Dr Conaghan, Department of Rheumatology, Leeds General Infirmary, Great George St, Leeds LS 1 3EX, UK

philip.conaghan@ leedsth.nhs.uk

Accepted 17 April 2002
A good understanding of osteoarthritis (OA) and its relation to exercise is essential for the sports doctor who is often faced with both the patient with established OA participating in sport and the patient with predisposing factors to the later development of OA. Increasing evidence from the last decade has strengthened the relation between occupational activities and the risk of developing and accelerating OA. Recent studies have also provided insight into the relation between exercise and OA. It is therefore timely to review these associations and evaluate current $\mathrm{OA}$ treatments with an emphasis on newer treatments.

When critically analysing the literature on OA associations, it is worth noting the study definition of OA-whether self reported or based on clinical and/or radiographic findings-as this will obviously influence incidence and progression findings. Obviously the nature of the study design (and there are many small cross sectional studies in the sports OA literature) is important in adding weight to a particular association. Much of this review pertains to OA of the knee, reflecting the predominance of knee studies in the OA literature. This review focuses on human studies rather than animal experiments, which have been reviewed elsewhere. ${ }^{1-3}$

\section{MODERN CONCEPTS OF OA}

$$
\begin{aligned}
& \text { "OA is best conceptualised not as an } \\
& \text { acquired disease but as an evolutionary } \\
& \text { process" }
\end{aligned}
$$

OA remains by far the most common disease of joints and represents a huge burden to society in terms of personal suffering and health resource utilisation. It is clearly associated with aging, of particular importance in the Western world with the aging of the baby boomer generations. ${ }^{4}$ It is best conceptualised not as an acquired disease but as an evolutionary process; this is well supported by research involving macaque monkeys who walk upright and develop OA in the same joints as man, except for the thumb, which they cannot oppose. $^{5}$ The common joints involved (cervical and lumbar spine, hip, knee, and base of thumb) are those involved with increased load and weight bearing. Dieppe ${ }^{6}$ has described three steps in the OA process: (a) initiation, either traumatic or in susceptible joints; $(b)$ the process, which is biochemically mediated but biomechanically driven; (c) clinical expression, which may be modified by factors such as weight and sex.

Although much research has focused on the role of cartilage in OA, it is clear that this is a disease of the whole joint organ; structures such as the synovium and subchondral bone are important in the disease process. ${ }^{4}$ Recent publications have highlighted the importance of inflammation in the OA process: positive bone scintigraphy, $\mathrm{C}$ reactive protein levels, and degree of arthroscopic synovitis $^{78}$ all predict progression of knee OA.

Pain is the main reason patients with OA will seek help. There has generally been a poor correlation between pain and structural damage (as evidenced by conventional radiographs). ${ }^{9}$ However, novel imaging modalities such as magnetic resonance imaging (MRI) that are tomographic and able to visualise soft tissue components of the joint are making important contributions to our understanding of OA. Recent findings from a large cohort study in Boston have shown that the site of patient pain correlates with the site of underlying MRI bone oedema, ${ }^{10}$ and that degree of pain correlates with degree of synovial hypertrophy. ${ }^{11}$ All these findings have important consequences for our understanding of disease pathogenesis as well as targeting of future treatments.

\section{RISK FACTORS FOR OA}

The risk factors for development of OA may be seen as general/systemic (genetic, age) or local/ biomechanical (obesity, trauma, knee alignment). ${ }^{4}$ Many of these risk factors have been linked with the incidence of radiographic OA rather than the progression, ${ }^{12}{ }^{13}$ supporting the concept of separate OA initiation and disease processes. The factors relevant to sport and exercise will be discussed below.

\section{Occupation}

Of analogous interest when considering the effects of load bearing exercise on joints are the

Abbreviations: OA, osteoarthritis; MRI, magnetic resonance imaging 
effects of occupation. Jobs that require heavy lifting together with high levels of squatting and kneeling are associated with high levels of hip and knee OA. ${ }^{13-15}$ In a large well documented cohort (Framingham), the number of hours of heavy physical activity has been linked to the risk of incident radiographic knee OA. ${ }^{13}$ There is an even greater risk of OA with heavy physical activity in the obese. ${ }^{13}{ }^{15}$ These risks are also present in small joints: textile mill workers using repetitive pincer grip develop high rates of distal interphalangeal joint OA. ${ }^{16}$ One of the confounding factors in interpreting many OA-sport associations has been the limited evidence gathered on participants' occupations.

\section{Joint injury}

Two recent, large, prospective cohort studies have reinforced the importance of injury as a risk factor for OA. In an American study of 1321 former medical students, ${ }^{17}$ mean entry age was 22 and median follow up was 36 years. Over the follow up period, 141 reported injuries of knee or hip, and 96 developed OA. By age 65 , those with childhood or adolescent injuries had a cumulative OA incidence of $13.9 \%$ compared with $6 \%$ in those without injury. The relative risks for developing knee and hip OA after injury were 5.17 and 3.5 respectively. A study from England of 354 patients over the age of 55 followed for a mean of 5.1 years also found previous knee injury to be associated with increased risk of knee OA (odds ratio 4.8). ${ }^{12}$

There is considerable overlap of studies involving sport, injury, and OA. The increased risks associated with injuries such as ligament tears and meniscal injuries in the knee and fractures involving articular surfaces have been appreciated for many years, and many of these studies were based on sports participants. ${ }^{18-22} \mathrm{~A}$ recent review of knee injuries pointed out that most studies have been retrospective with varying duration of follow up. ${ }^{22}$ These studies showed that isolated anterior cruciate ligament or meniscal injuries with repair are associated with a 10-fold increase in OA, meniscectomy with intact ligaments further doubles that risk, and a combination of injuries results in much higher long term incidence of radiographic OA.

In view of the association of injury, sports, and OA, and with prevention strategies in mind, it is appropriate to see detailed data emerging on the incidence of injuries in different sports. For example, elite soccer players (from four English league clubs) were studied prospectively over four years using physiotherapists to record injuries. ${ }^{23}$ This study showed an injury incidence of 8.5 per 1000 hours; over a fifth of these were re-injuries. Another report of female adolescent soccer players from Sweden followed over one season showed an injury rate of 6.8 per 1000 hours. ${ }^{24}$ Both studies showed much higher injury rates during games than during training.

Sensitive imaging methods have also improved our knowledge of injury-OA associations. Using high definition macroradiographs, Buckland-Wright et al ${ }^{25}$ showed considerable thickening in the subchondral bone trabeculae of subjects with ruptured anterior cruciate ligament only four years after injury. Osteophytes were present in about half of these knees, but joint space narrowing was not seen. Another report using MRI six years after anterior cruciate ligament rupture found cartilage thinning in a significant number of patients and persistent evidence of altered bone marrow signal (or bone oedema) in over half the subjects. ${ }^{26}$

\section{EXERCISE AND OA}

The association between sport and OA has not always been clear because of the different natures of the sports involved (with different levels of injury), different levels of subject participation, the confounding effects of occupation (usually not recorded), and, most importantly, the design of the studies. An excellent review by Lequesne et al has comprehensively summarised the relevant studies according to study design. The current review will attempt to update and classify the literature into two broad categories of OA: studies on high levels of sport involvement, including elite athletes, and those on low to moderate levels of sport involvement, including recreational sport activities.

\section{High levels of sports participation}

There does seem to be a strong association between elite sports participation and an increased risk of OA. However, the nature of the sport is very important to the degree of risk. The sports with major risk are those that involve repetitive, high intensity, high impact forces through the affected joints, especially where there is a high associated risk of injury.

A Finnish group has presented a number of interesting studies comparing former international level competitors. They looked at hospital admission rates over 21 years for OA (hip, knee, or ankle) for over 2000 male ex-athletes. ${ }^{27}$ They divided the cohort into endurance (long distance running, cross-country skiing), team (soccer, ice hockey, basketball, track, and field), and power (boxing, wrestling, weightlifting, throwing) sports. All three groups had higher incidences of OA related admissions, with power and team sports having admissions at an earlier age. Subsequently they reported on 117 of these athletes chosen for different knee loading sports. ${ }^{28}$ The prevalence of radiographic OA knee for long distance running, soccer, weightlifting, and shooting were $14 \%$, $29 \%, 31 \%$, and $3 \%$. Soccer players and weightlifters had higher risk which was due, at least partly, to knee injuries in the former and high body mass index at age 20 in the latter. Recently this group presented data on hip and knee disability, rather than OA rates. ${ }^{29}$ The former power sport competitors had the highest odds ratios for hip disability, whereas only the team sport players had a high risk for knee disability, again perhaps reflecting the injury prone nature of these sports.

Elite soccer players are one of the groups most often studied, with an increasing number of reports adding to the evidence on the risks of this sport..$^{18}{ }^{28}{ }^{30-36} \mathrm{~A}$ Swedish retrospective cohort reported both hip and knee radiographic OA associations in 71 elite and 215 non-elite players with a mean age of 55 and compared the rates with those of 572 age matched controls. ${ }^{31}{ }^{32}$ For the hip, the prevalence of OA was $14 \%$ for elite players compared with $4.2 \%$ in non-elite and control groups. ${ }^{31}$ For the knee, the prevalence of OA was $15.5 \%, 4.2 \%$, and $1.6 \%$ in elite, non-elite, and control groups. ${ }^{32}$ Two recent British surveys looked at lower limb OA in large numbers of ex-professional soccer players. ${ }^{35}{ }^{36}$ These studies reported rates of lower limb OA of $49 \%^{35}$ and $32 \%{ }^{36}$ of these former players. The knee was the most common site. Another code of football, Australian Rules, has also been shown to involve greater risks of clinical and radiographic OA knee than controls in a retrospective cohort study; yet again injury increased the risk. ${ }^{37}$

The data from studies of runners have been a little more difficult to understand but it may be that the risks for OA depend on the amount and rate of mileage run. In a retrospective cohort study, examinations were performed in 1973 and 1988 on a number of former athletes: 27 long distance runners (averaging 60 miles/week in 1973), nine bobsleigh riders, and 23 controls. $^{38}$ The athletes had a mean age of 42 years at the second examination. Radiographic OA hip was found in 19\% of the runners, but in neither of the other groups. Age and number of miles run per week in 1973 were the positive predictors of radiographic OA. Spector et al ${ }^{39}$ compared 67 female elite middle and long distance runners and 14 female tennis players (aged 40-65) with a large matched control group. ${ }^{39}$ Radiographic hip and knee OA rates were significantly higher in the former athletes, with a tendency to more patellofemoral OA in the runners. There are a number of negative association studies but design problems are clear. In a retrospective cohort study, no higher rates of radiographic OA were seen in 60 ex-marathon runners than in controls, although the timing of radiographs differed for the 
two groups. ${ }^{40}$ A study comparing 504 university level cross country runners with similar level swimmers found no difference in levels of hip and knee pain but $x$ ray examinations were not performed. ${ }^{41}$ A small prospective study of 17 male runners (nine were marathon runners) compared with controls found no difference in radiographic OA at the hip, knee, ankle, or feet. ${ }^{42}$

Lane and colleagues ${ }^{43-45}$ have presented a series of reports from a well described, prospective cohort of subjects from a long distance running club-perhaps closer to recreational runners than the elite athletes studied above. At baseline, 41 runners aged 50-72 years and averaging 25 miles a week were compared with controls matched for age, sex, years of education, and occupation. ${ }^{43}$ There was no difference in clinical and radiographic OA findings in the knee and lumbar spine. Follow up of this cohort at five ${ }^{44}$ and nine ${ }^{45}$ years showed similar rates of progression for radiographic OA of the knee in the runners and controls with no significant between group differences. For the runners, regression analysis disclosed that the predictors of progression of radiographic knee OA were baseline radiographic score and a faster pace per mile. Hip radiographs taken after nine years also showed no differences in OA between the groups. ${ }^{45}$ Another study of 30 long distance runners, 90\% of whom averaged 12.5-25 miles a week over a median of 40 years, found no differences in rates of radiographic OA at the hips, knees, and ankles when compared with controls matched for age, body mass index, and occupation. ${ }^{46}$

\section{Low to moderate levels of sports participation}

Physical education teachers have been studied to assess the effects of moderate exercise. A report of 364 women who trained as such teachers showed no difference in radiographic OA of hip and knees compared with historical, population based controls. ${ }^{47}$ Another large study of female physical education teachers compared with population based controls found no increase in rates of radiographic hip or knee OA, and actually found lower rates of knee OA. ${ }^{48}$ Different findings were presented in a recent study of 571 graduates from the single Swedish training facility. ${ }^{49}$ The graduates had a current median age of about 57 and were matched with people from a population register. Both male and female teachers had higher rates of self reported OA knee and knee injury than the comparator group. However, radiographs were not taken in this study and therefore the prevalence of radiographic OA is not certain. It is also difficult to compare the extent of sporting participation in these reports.

Various population based studies have studied the effects of sport on OA rates. Such studies are likely to present a picture of overall associations, but it should be remembered that there is a wide variation in amount of sporting participation and that these studies use recalled levels of sporting activities. Cooper et $a l^{12}$ examined a large cohort with knee radiographs taken five years apart and median age at follow up of 75.8 years. They found that a history of regular sport participation was associated with an increased risk of incident (but not progression of) OA knee. Vingard et al presented results on the risks for hip joint replacement (a marker for symptomatic and operable OA but presumably a subset of the radiographic OA population) from retrospective case-control studies of Swedish male ${ }^{50}$ and female ${ }^{51}$ population based cohorts. For those with the most hours of recalled sporting activities (over 800 hours in total based on recall of all sporting involvement up till the age of 50 ), men had a relative risk of 4.5 and women 2.3 of developing OA of the hip, compared with the lowest activity groups. Importantly men who had performed jobs with high joint loading as well as high levels of sport had almost double the relative risk. Lane $e^{2}$ al $^{52}$ used a regression analysis to examine the association of reported exercise and radiographic OA hip in a population of 5818 women with a mean age of about 72 . Women who reported regular recreational physical activities (defined as 1 to

\section{Take home message}

- Joint injury appears to be the strongest predictor for later development of OA

- Sports involving repetitive, high impact joint loading may also increase risk

- Risk assessment must include risk factors for OA such as body mass index and occupation

5 times a week) as teenagers, at age 30 , and at age 50 had significantly greater odds of moderate to severe hip OA than those who performed no recreational activities.

\section{"The only strong risk factor for knee OA was a reported prior knee injury"}

Yet again, a major confounding issue has been separating the impact loading aspects of the sport from the associated injuries. A recent retrospective, case-control study from the United Kingdom used 216 subjects (drawn from a large national survey) with self reported OA knee and matched each of them with four controls. ${ }^{53}$ Extensive data on lifetime exercise involvement was obtained. The only strong risk factor for knee OA was a reported prior knee injury (odds ratio 8.0); most of these knee injuries were related to sport.

\section{MECHANISMS FOR SPORT ASSOCIATED OA}

The mechanism by which repetitive, high weight loading exercise causes $\mathrm{OA}$ is unclear. Bone remodelling with osteophyte formation is one of the early associated radiographic features, ${ }^{39} 44$ and this may precede cartilage degeneration. As mentioned above, MRI will have a large impact on our understanding of joint pathology related to exercise. The ability of MRI to simultaneously image soft tissue, bone, and cartilage as well as its tomographic properties suggests that it may be possible to identify soon after injury those subjects who are at greatest long term risk for OA. A preliminary study on eight non-elite runners investigated before and eight weeks after a marathon competition showed that only the single runner with pre-existing meniscal damage had signs of progressive degenerative change ${ }^{54}$; such findings would be consistent with many of the radiographic studies listed above. Although the role of biomarkers as indicators of OA progression is not yet clear, ${ }^{55}$ they may also provide insights into the structures involved in early joint damage. A recent study, again from eight marathon runners, showed that levels of cartilage oligomeric matrix protein (a marker of cartilage turnover and destruction) were raised at baseline (perhaps reflecting training) and increased during the marathon. ${ }^{56}$ The clinical significance of these findings is as yet unclear.

\section{BENEFITS OF EXERCISE}

It is important not to see the possible deleterious effects of sport in isolation-the benefits to the body as a whole may still outweigh local problems. A number of the studies already cited have shown such benefits. These benefits may be present in both elite and recreational athletes. Sarna $e t$ a $l^{57}$ have reported on morbidity and mortality drawn from national registries for a very large cohort of Finnish male elite athletes compared with population controls. This study showed that participants in aerobic sport (the endurance and team sport categories listed above) had high life expectancy and low risks for ischaemic heart disease and diabetes. A study of physical education teachers reported less cigarette smoking, fewer serious diseases, and less obesity in the teachers than a control population. ${ }^{49}$ Lane et $a l^{58}$ found that 498 runners compared with population controls had better cardiovascular fitness and reduced obesity. The runners also used medical services less often. 


\section{SUMMARY}

Overall it appears reasonable to conclude from the literature that there is an increased risk of lower limb OA associated with repetitive, high impact sports and that this risk is strongly associated with joint injury. The data from population studies suggest increased risk for OA, although such studies cannot give indications of which sports may be less harmful to joints. There seems to be little risk associated with recreational running. The general health benefits to be gained from exercise remain very important. When assessing an athlete's risk for OA, the nature of the sport, intensity of training, presence of previous injury, body mass index, and occupation should all be taken into account.

\section{REFERENCES}

1 Buckwalter JA. Osteoarthritis and articular cartilage use, disuse, and abuse: experimental studies. J Rheumatol 1995;22(suppl): 13-5.

2 Lequesne MG, Dang N, Lane NE. Sport practice and osteoarthritis of the limbs. Osteoarthritis Cartilage 1997;5:75-86.

3 Van den Berg WB. Lessons from animal models of osteoarthritis. Curr Opin Rheumatol 2001;13:452-6.

4 Felson DT, Lawrence RC, Dieppe PA, et al. Osteoarthritis: new insights. Part 1. The disease and its risk factors. Ann Intern Med 2000;133:635-46.

5 Lim K, Rogers J, Shepstone L, et al. The evolutionary origins of osteoarthritis: a comparative study of hand disease in two primates. $J$ Rheumatol 1995;22:2132-4.

6 Dieppe P. Osteoarthritis and molecular markers. A rheumatologist's perspective. Acta Orthop Scand 1995;66(suppl): 1-5.

7 Kirwan JR, Elson CJ. Is the progression of osteoarthritis phasic? Evidence and implications. J Rheumatol 2000;27:834-6.

8 Ayral X, Ravaud P, Bonvarlet JP, et al. Arthroscopic evaluation of post-traumatic patellofemoral chondropathy. J Rheumatol 1999;26: 11 140-7.

9 Hannan MT, Felson DT, Pincus T. Analysis of the discordance between radiographic changes and knee pain in osteoarthritis of the knee. $J$ Rheumatol 2000;27:1513-17

10 Felson DT, Chaisson CE, Hill CL, et al. The association of bone marrow lesions with pain in knee osteoarthritis. Ann Intern Med 2001;134:541-9.

11 Hill CL, Gale DG, Chaisson CE, et al. Knee effusions, popliteal cysts, and synovial thickening: association with knee pain in osteoarthritis. $J$ Rheumatol 2001;28:1330-7.

12 Cooper C, Snow S, McAlindon TE, et al. Risk factors for the incidence and progression of radiographic knee osteoarthritis. Arthritis Rheum 2000;43:995-1000.

13 McAlindon TE, Wilson PWF, Aliabadi P, et al. Level of physical activity and the risk of radiographic and symptomatic knee osteoarthritis in the elderly: the Framingham study. Am J Med 1999;106:151-7.

14 Maetzel A, Makela M, Hawker G, et al. Osteoarthritis of the hip and knee and mechanical occupational exposure: a systematic overview of the evidence. J Rheumatol 1997;24:1599-607

15 Coggon D, Croft $P$, Kellingray S, et al. Occupational physical activities and osteoarthritis of the knee. Arthritis Rheum 2000;43:1443-9.

16 Hadler NM, Gillings DB, Imbus HR, et al. Hand structure and function in an industrial setting. Influence of three types of stereotyped repetitive usage. Arthritis Rheum 1978;21:210-20

17 Gelber AC, Hochberg MC, Mead LA, et al. Joint injury in young adults and risk for subsequent knee and hip osteoarthritis. Ann Intern Med 2000;133:321-8.

18 Neyret P, Donell ST, DeJour D, et al. Partial meniscectomy and anterior cruciate ligament rupture in soccer players: a study with a minimum 20 years follow up. Am J Sports Med 1993;21:455-60.

19 Honkonen SE. Degenerative arthritis after tibial plateau fractures. $J$ Orthop Trauma 1995:9:273-7.

20 Lundberg $\mathbf{M}$, Messner K. Ten-year prognosis of isolated and combined medial collateral ligament ruptures. A matched comparison in 40 patients using clinical and radiographic evaluations. Am J Sports Med 1997;25:2-6.

21 Roos $\mathbf{H}$, Lauren $M$, Adalberth $T$, et al. Knee osteoarthritis after meniscectomy: prevalence of radiographic changes after twenty-one years, compared with matched controls. Arthritis Rheum 1998;41:687-93.

22 Gillquist J, Messner K. Anterior cruciate ligament reconstruction and the long-term incidence of gonarthrosis. Sports Med 1999;27:143-56.

23 Hawkins RD, Fuller CW. A prospective epidemiological study of injuries in four English professional football clubs. Br J Sports Med 1999;33:196-203

24 Soderman K, Adolphson J, Lorentzon R, et al. Injuries in adolescent female players in European football: a prospective study over one outdoor season. Scand J Med Sports 2001;11:299-304.

25 Buckland-Wright JC, Lynch JA, Dave B. Early radiographic features in patients with anterior cruciate ligament rupture. Ann Rheum Dis 2000;59:641-6.
26 Faber KJ, Dill JR, Amendola A, et al. Occult osteochondral lesions after anterior cruciate ligament rupture. Six year magnetic resonance imaging follow-up study. Am J Sports Med 1999;27:489-94

27 Kujala UM, Kaprio J, Sarna S. Osteoarthritis of weight-bearing joints of lower limbs in former elite male athletes. BM 1994;308:231-4.

28 Kujala UM, Kettunen J, Paananen $\mathrm{H}$, et al. Knee osteoarthritis in former runners, soccer players, weight lifters, and shooters. Arthritis Rheum 1995;38:539-46

29 Kettunen JA, Kujala UM, Kaprio J, et al. Lower-limb function among former elite male athletes. Am J Sports Med 2001;29:2-8.

30 Klunder KB, Rud B, Hansen J. Osteoarthritis of the hip and knee joint in retired football players. Acta Orthop Scand 1980;51:925-7.

31 Lindberg H, Roos H, Gardsell P. Prevalence of coxarthrosis in former soccer players. 286 players compared with matched controls. Acta Orthop Scand 1993;64:165-7.

32 Roos H, Lindberg H, Gardsell P, et al. The prevalence of gonarthrosis and its relation to meniscectomy in former soccer players. Am J Sports Med 1994;22:219-22.

33 Hawkins RD, Fuller CW. An examination of the frequency and severity of injuries and incidents at three levels of professional football. Br J Sports Med 1998;32:326-32.

34 Larsen E, Jensen PK, Jensen PR. Long-term outcome of knee and ankle injuries in elite football. Scand J Med Sci Sports 1999;9:285-9.

35 Turner AP, Barlow JH, Heathcote-Elliott C. Long term health impact of playing professional football in the United Kingdom. Br J Sports Med 2000;34:332-7.

36 Drawer S, Fuller CW. Propensity for osteoarthritis and lower limb joint pain in retired professional soccer players. Br J Sports Med $2001 ; 35: 402-8$

37 Deacon A, Bennell K, Kiss ZS, et al. Osteoarthritis of the knee in retired elite Australian Rules footballers. Med J Aust 1997;166:187-90.

38 Marti B, Knobloch M, Tschopp A, et al. Is excessive running predictive of degenerative hip disease? Controlled study of former elite athletes. BM 1989;299:91-3.

39 Spector TD, Harris PA, Hart DJ, et al. Risk of osteoarthritis associated with long-term weight-bearing sports. A radiological survey of the hips and knees in female ex-athletes and population controls. Arthritis Rheum 1996;39:988-95.

40 Puranen J, Ala-Ketola L, Peltokallio P, et al. Running and primary osteoarthritis of the hip. BMU 1975;2:424-5.

41 Sohn RS, Micheli $\mathrm{L}$. The effect of running on the pathogenesis of osteoarthritis of the hips and knees. Clin Orthop Res 1985;198:106-9.

42 Panush RS, Schmidt C, Caldwell JR, et al. Is running associated with degenerative joint disease? JAMA 1986;255:1152-4.

43 Lane NE, Bloch DA, Jones $\mathrm{HH}$, et al. Long-distance running, bone density, and osteoarthritis. JAMA 1986;255:1147-51.

44 Lane NE, Michel B, Bjorkengren A, et al. The risk of osteoarthritis with running and aging: a 5-year longitudinal study. J Rheumatol 1993;20:461-8.

45 Lane NE, Oehlert JW, Bloch DA, et al. The relationship of running to osteoarthritis of the knee and hip and bone mineral density of the lumbar spine: a 9 year longitudinal study. J Rheumatol 1998;25:334-41.

46 Konradsen L, Hansen EM, Sondergaard L. Long distance running and osteoarthrosis. Am J Sports Med 1990;18:379-81.

47 Eastmond CJ, Hudson A, Wright V. A radiological survey of the hips and knees in female specialist teachers of physical education. Scand Rheumatol 1979;8:264-8.

48 White JA, Wright V, Hudson AM. Relationships between habitual physical activity and osteoarthrosis in aging women. Public Health 1993:107:459-70.

49 Sandmark H. Musculoskeletal dysfunction in physical education teachers. Occup Environ Med 2000;57:673-7.

50 Vingard E, Alfredsson L, Goldie I, et al. Sports and osteoarthrosis of the hip. An epidemiologic study. Am J Sports Med 1993;21:195-200.

51 Vingard $\mathbf{E}$, Alfredsson L, Malchau $\mathrm{H}$. Osteoarthrosis of the hip in women and its relationship to physical load from sports. Am J Sports Med 1998;26:78-82.

52 Lane NE, Hochberg MC, Pressman A, et al. Recreational physical activity and the risk of osteoarthritis of the hip in elderly women. $J$ Rheumatol 1999;26:841-54.

53 Sutton AJ, Muir KR, Mockett S, et al. A case-control study to investigate the relation between low and moderate levels of physical activity and osteoarthritis of the knee using data collected as part of the Allied Dunbar National Fitness Survey. Ann Rheum Dis 2001;60:756-64.

54 Krampla W, Mayrhofer R, Malcher J, et al. MR imaging of the knee in marathon runners before and aft competition. Skeletal Radiol $2001 ; 30: 72-6$

55 Garnero P, Rousseau JC, Delmas PD. Molecular basis and clinical use of biochemical markers of bone, cartilage, and synovium in joint diseases. Arthritis Rheum 2000;43:953-68.

56 Neidhart M, Muller-Ladner U, Frey W, et al. Increased serum levels of non-collagenous matrix proteins (cartilage oligomeric matrix protein and melanoma inhibitory activity) in marathon runners. Osteoarthritis Cartilage 2000;8:222-9.

57 Sarna S, Kaprio J, Kujala UM, et al. Health status of former elite athletes. Aging (Milano) 1997;9:35-41.

58 Lane NE, Bloch DA, Wood PD, et al. Aging, long-distance running, and the development of musculoskeletal disability. A controlled study. Am J Med 1987:82:772-8. 